УДК 517.9; 004; 551.58

\title{
ИССЛЕДОВАНИЕ ИЗМЕНЕНИЙ ГЛОБАЛЬНОГО КЛИМАТА КАК СЛОЖНОЙ СИСТЕМЫ С ИСПОЛЬЗОВАНИЕМ ВЕЙВЛЕТНЫХ ФАЗО-ЧАСТОТНЫХ ФУНКЦИЙ, ФАЗО-ЧАСТОТНЫХ И ФАЗО-ВРЕМЕННЫХ ХАРАКТЕРИСТИК ГЕЛИОКОСМИЧЕСКИХ И КЛИМАТИЧЕСКИХ ПЕРЕМЕНHЫХ. ЧАСТЬ 1
}

\author{
Алексеев Валерий Иванович, \\ v_alekseev@ugrasu.ru \\ Югорский государственный университет, \\ Россия, 628012, г. Ханты-Мансийск, ул. Чехова, 16
}

\begin{abstract}
Актуальность исследования обусловлена необходимостью установления истинных причин и закономерностей изменений глобального климата на Земле как сложной системы.

Цель: разработать и предложить эфффективный метод извлечения информации об изменчивости переменных, содержащейся в исходных данных изменений гелиокосмических и климатических переменных, заданных в виде временных рядов, и применить этот метод для обоснования климатических изменений на Земле.

объекты: временные ряды изменений гелиокосмических и климатических переменных.

Методы: непрерывное прямое вейвлет-преобразование исходных данных с использованием комплексного вейвлета 'cgaи5' c вычислением их временных вейвлетных фразо-частотных функций, фразо-частотных и фразо-временных характеристик; оценка спектра периодичностей фрункций по фразо-временным характеристикам переменных; оценка и анализ плотностей распределений фазо-частотных и фазо-временных характеристик; оценка и анализ коэффиицентов корреляций между фазочастотными и фразо-временными характеристиками переменных в равных интервалах времени.

Результаты. Получены временные вейвлетные фазо-частотные функции и их изображения, позволяющие визуализировать и оценить спектры периодичностей исследуемых переменных; оценки вейвлетных фазо-частотных и фазо-временных характеристик переменных, позволяющие проводить весь комплекс сравнительных исследований переменных и установить закономерности их изменений. К этим исследованиям относятся: оценивание плотностей распределений этих характеристик, позволяющих идентифицировать фракторы, влияющие на эти изменения; вычисление матриц корреляций между вейвлетными фразо-частотными и фразо-временными характеристиками переменных в заданных равных интервалах времени, позволяющие оценить степени согласованности изменений переменных в фазо-частотной области и в области фразовременных изменений. Установлена тесная взаимосвязь между колебательными процессами гелиокосмических переменных, солнечной активностью и вулканическими процессами на Земле, изменениями концентрации двуокиси углерода $\mathrm{CO}_{2}$ в атмоссрере. Показано, что движение Солнца относительно барицентра, обусловленное движением планет-гигантов Солнечной системы, управляет солнечной активностью, тектоническими процессами на Земле, в том числе вулканическими извержениями, климатом на Земле.
\end{abstract}

\section{Ключевые слова:}

Глобальное изменение климата, непрерывное прямое вейвлет-преобразование, временная вейвлетная фразо-частотная функция, вейвлетная фазо-частотная и фазо-временная характеристики, коэффициенты корреляций.

\section{Введение}

По данным Всемирной метеорологической организации (ВМО) 20 самых теплых лет в истории наблюдений приходятся на последние 22 года, а четыре самых теплых года - на четыре последних года $[1,2]$. В работах подчеркивается, что главным фактором роста приземной температуры в современности является рост концентрации в атмосфере парниковых газов, углекислого газа $\mathrm{CO}_{2}$, выбрасываемого в атмосферу человечеством при использовании ресурсов ископаемого топлива. В итогах конференции по изменению климата в Катовице (Польша) СОП24 в 2018 г. [3], в докладе об особенностях климата на территории Российской Федерации за 2018 [4], в докладе о климатических рисках на территории Российской Федерации [5], а также в работах [6-10] утверждается, что главным поставщиком парниковых газов в атмосферу является человеческая деятельност.

В коллективных научных трудах [11-13] представлены фундаментальные научные исследования о факторах, определяющих современные глобальные изменения природной среды и глобального климата; о влиянии гелиокосмических, геофизических и других процессов на развитие вулканизма и изменении глобального климата. На изменения природной среды влияют многие усиливающиеся в современности факторы, в том числе и воздействие деятельности человека.

Изменения природной среды и климата во времени и в пространстве обусловлены изменениями факторов, влияющих на эти процессы: гелиокосмических, геофизических, климатических и антропогенных.

Цель исследования - разработка метода, позволяющего установить закономерности изменений глобального климата в прошлом, в современности и причины этих изменений.

\section{Исходные данные}

Решение задачи производится анализом гелиокосмических переменных и многих других факторов, влияющих на природные процессы Земли [11-13]. В качестве таких факторов использованы графики изменений барицентрического движения Солнца, асимметричного движения Солнца вокруг центра 
масс (ЦМ), относительных отклонений угловой скорости вращения Земли от эталонной величины, отклонения длительности суток, расстояния от ЦМ сол- нечной системы до Солнца и до Земли. Графики изменений этих и других переменных, использованных в работе, приведены на рис. 1.

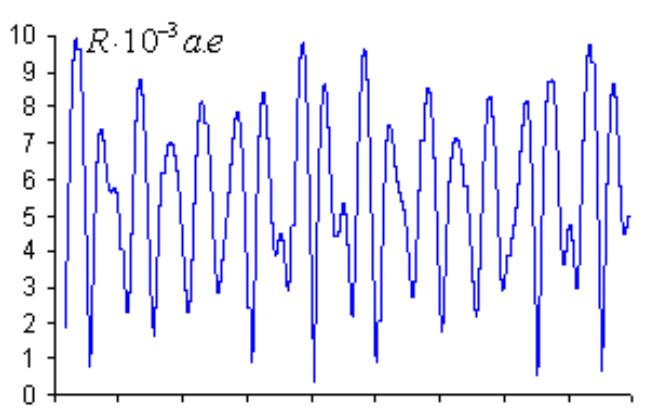

$1650169017301770181018501890193019702 n 10$

a)

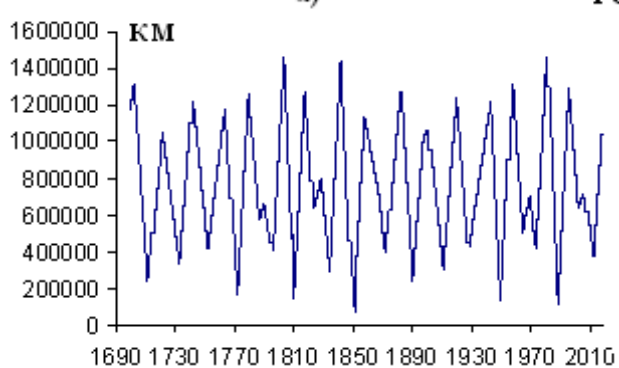

годь]

c)

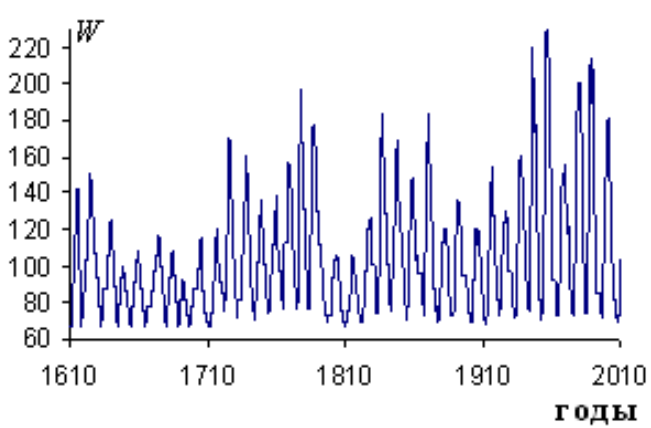

e)

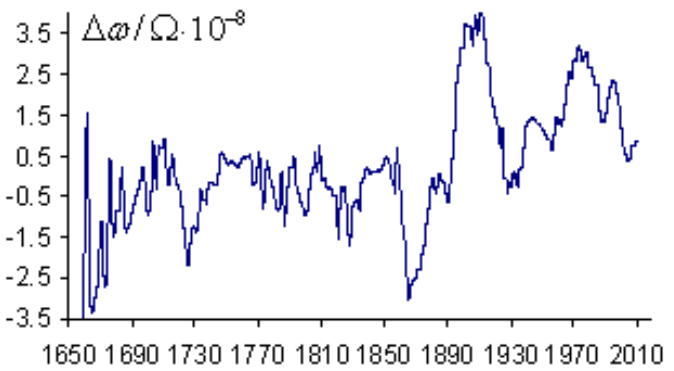

g)

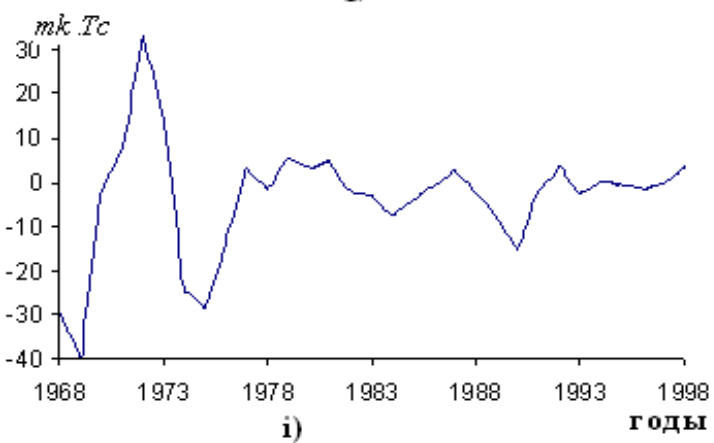

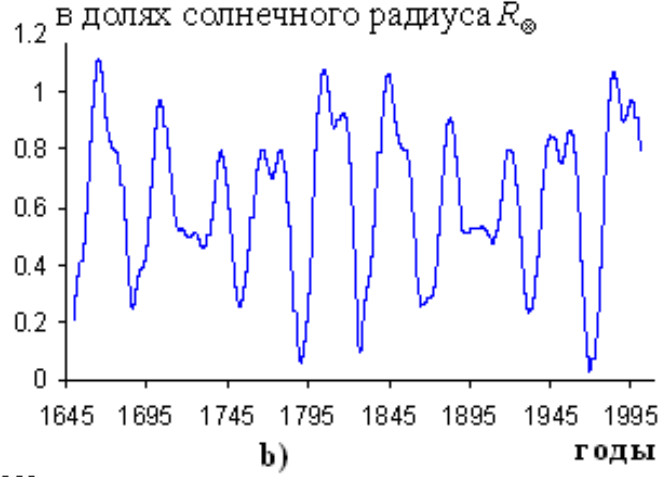

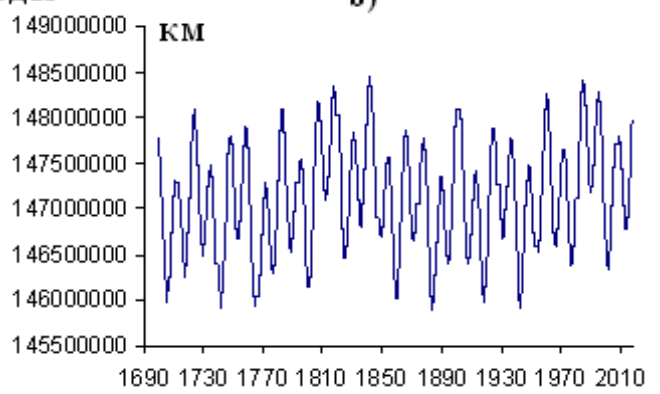

d)

годы

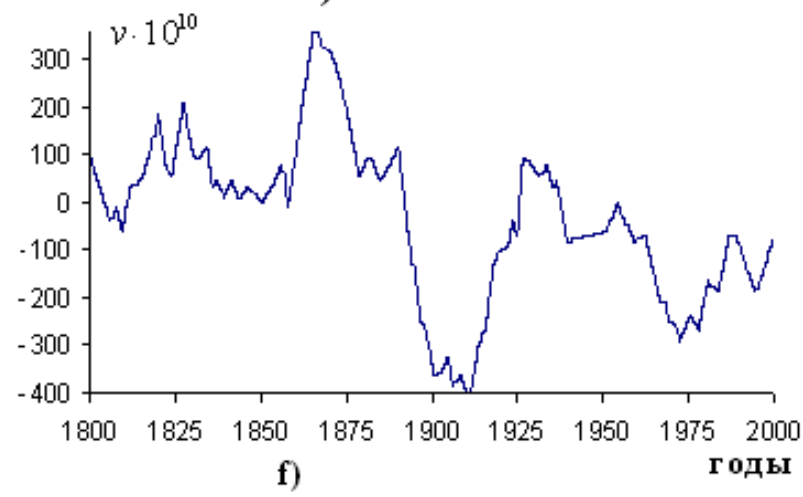

а-индекс гемагнитной активности

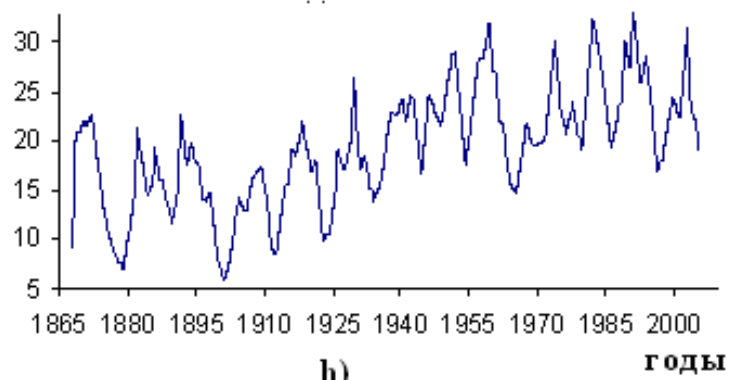

h)

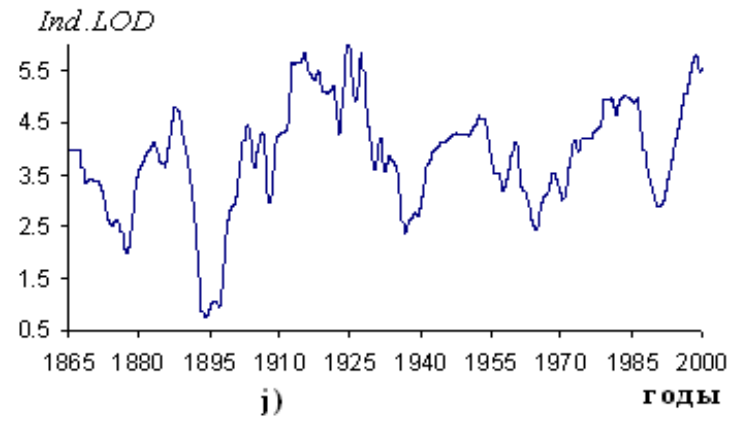





Рис. 1. Графики изменений гелиокосмических, климатических факторов: a) $f_{1}(t)$ - изменение со временем расстояния иентта масс (ЦМ) Солниа от барицентра в $10^{-3}$ a.е. по ординате на интервале с 1656 до 2009 г2. с учетом Юпитера, Сатурна, Урана и Нептуна [13]; b) $f_{2}(t)$ - асимметричное движение Солниа вокруг ЦМ солнечной системы, расстояние Солнца от субъюпитерной точки (в долях солнечного радиуса по ординате) по ординате в 1650-2000 гг. [14]; c) $f_{3}(t)$ - изменение расстояния ЦМ солнечной системы до Солниа в км в 1700-2018 г2. [15]; d) $f_{4}(t)$ - изменение расстояния ЦМ солнечной системы до Земли в км в 1700-2018 г2. [15]; e) $f_{5}(t)$ - временные вариации солнечной активности (чисел Вольфа по ординате) в 1700-2010 г2. [16]; f) $f_{6}(t)$ - ход изменения скорости вращения Земли. По оси ординат отложены относительные уклонения угловой скорости $v \cdot 10^{10}$ в 1610-2012 ге. [17]; g) $f_{7}(t)$ - ход среднегодовых относительных отклонений угловой 
скорости вращения Земли от эталонной величины $\left(\Delta \omega / \Omega \cdot 10^{-8}\right)$ по ординате в интервале с 1656 по 2008 г2. [13]; h) $f_{8}(t)$ - изменение индекса «а» геомагнитной активности Земли в 1868-2000 г2. (в магнитных индексах) [18]; i) $f_{9}(t)$ - временные вариации среднегодовых значений напряженности общего магнитного поля Солниа в 1968-2000 г2. (в микротеслах) [19], j) $f_{10}(t)$ - график скорости изменения со временем длительности земных суток (LOD) между 1865 и 2005 г2., скользящие средние по пяти годам [14]; $k) f_{11}(t)$ - график количества вулканических извержений в мире в 1610-2014 г2. [20]; l) $f_{12}(t)$ - график количества зарегистрированных иунами в мире в 1820-2006 г2. [21]; $m) f_{13}(t)$ - изменение количества землетрясений в мире в 1900-2010 г2. с усреднением по пяти годам [21]; n) $f_{14}(t)$ - количество зарегистрированных катастроф в Мире в 19002010 г2. [22]; o) $f_{15}(t)$ - график изменения аномалии средней глобальной приземной температуры в 1850-2009 г2. относительно среднего в 1961-1990 г2. [23]; p) $f_{16}(t)$ - график изменения конщентрации $\mathrm{CO}_{2}$ в ррт в атмосфере Земли в 1610-2012 г2. [23]; q) $f_{17}(t)$ - график изменения индекса Североатлантического колебания (NAO) между 1864 и 2006 г2., скользящие средние по пяти годам [14, 24]; r) $f_{18}(t)$ - график изменения индекса Тихоокеанского декадного колебания (PDO) в 1707-2000 г2. [14]; s) $f_{19}(t)$ - график изменения аномалии глобальной температуры воздуха $\Delta t{ }^{\circ} \mathrm{C}$ в районе суши между 1850 и 2005 г2. [18]; $\left.t\right) f_{20}(t)$ - график изменения аномалии глобальной температуры воздуха $\Delta t{ }^{\circ} \mathrm{C}$ на поверхности Мирового океана между 1850 и 2005 г2. [18]

Fig. 1. Graphs of changes in solar and climatic factors: a) change over time of the distance of the Sun center of mass (CM) from the barycenter in a.u. by ordinate in the interval from 1656 to 2009, taking into account Jupiter, Saturn, Uranus and Neptune [13]; b) the asymmetric movement of the Sun around the solar center of the solar system, the distance of the Sun from the sub-pit point (in fractions of the solar radius along the ordinate) along the ordinate in 1650-2000 [14]; c) the change in the distance of the solar system to the Sun in km in 1700-2018 [15]; d) change in the distance of the CM of the solar system to the Earth in $\mathrm{km}$ in 1700-2018 [15]; e) temporary variations in solar activity (Wolf numbers in ordinate) in 1700-2010 [16]; f) is the course of the change in the Earth rotation speed. The relative deviations of the angular velocity in 1610-2012 are plotted along the ordinate axis [17]; $g$ ) the course of the average annual relative deviations of the angular velocity of the Earth's rotation from the reference value along the ordinate in the interval from 1656 to 2008 [13]; h) change in the index «a» of the geomagnetic activity of the Earth in 18682000 (in magnetic indices) [18]; i) temporal variations of the annual average values of the total magnetic field of the Sun in 1968-2000 (in microtesla) [19]; j) graph of the rate of change with time of the duration of the Earth's Day (LOD) between 1865 and 2005, moving averages over five years [14]; $k$ ) graph of the number of volcanic eruptions in the world in 1610-2014 [20]; l) graph of the number of registered tsunamis in the world in 1820-2006 [21]; m) change in the number of earthquakes in the world in 1900-2010 with averaging over five years [21]; n) the number of recorded disasters in the World in 1900-2010 [22]; o) graph of changes in the anomaly of the average global surface temperature in 1850-2009 relative to the average in 1961-1990 [23]; p) graph of changes in concentration in the atmosphere of the Earth in 1610-2012 [23]; q) graph of changes in the North Atlantic Oscillation (NAO) Index between 1864 and 2006, moving averages over five years [14, 24]; r) graph of the Pacific Decade Oscillation Index (PDO) in 1707-2000 [14]; s) graph of changes in the anomaly of global air temperature in the land area between 1850 and 2005 [18]; t) graph of changes in the anomaly of global air temperature on the surface of the oceans between 1850 and 2005 [18]

В изменениях приведенных выше факторов (функций) существует глубокая связь. Изменения каждой из этих функций в той или мере обусловлены изменениями других переменных. Для установления причинно-следственных связей в изменениях переменных необходимы инструменты, позволяющие установить эти связи. Таким инструментом, среди известных, выбран анализ временных вейвлетных фазо-частотных функций исследуемых переменных $[25,26]$ с вычислением их фазо-частотных и фазовременных характеристик. Это связано с тем, что в космическом пространстве, где действует закон всемирного тяготения масс И. Ньютона и законы движения планет И. Кеплера, движения в пространстве одной планеты или группы планет влияют не только на траекторию движений других планет, но и на процессы, происходящие внутри планет, которые улавливаются вейвлетными преобразованиями.

Вычисление временной вейвлетной фазо-частотной функции сигнала $y(t)=f(t)$ и его основных отличительных характеристик

В основе метода лежит вейвлет-преобразование [27-29]

$$
W f(a, b)=\frac{1}{\sqrt{a}} \int_{R} f(t) \cdot \psi\left(\frac{\overline{t-b}}{a}\right) \cdot d t .
$$

В исследованиях весьма полезно использование изменений мгновенных фазовых характеристик функции $f(t)$ во времени, вычисленных на множестве задаваемых исследователем масштабов $a(1: k)$. Это возможно при использовании вейвлетного преобразования Wf(a,b). Мгновенные фазовые характеристики функции $f(t)$ в пакете Matlab вычисляются по формуле $\varphi_{f}(a, b)=a n g l e[W(a, b)]$, изменяющиеся в интервале $\pm \pi$ радиан, где комплекснозначная матрица $W f(a, b)$ размерности $a \times N, N$ - число дискретных отсчетов сигнала $f(t)$ в исследуемом временном интервале. В формуле (1) функция $\psi \overline{\left(\frac{t-b}{a}\right)}-$ комплексносопряженный вейвлет с параметрами $a(1: k)$ и $b$, где $b$ - параметр сдвига на временной оси (в вычислениях $b$ изменяется в интервале $1: N)$; максимальное значение $k$ масштаба $a$ вейвлета определяется частотным составом, интервалом времени наблюдения сигнала $f(t)$. Например, для наблюдений факторов более 300 лет $k=1000$, для наблюдений около 150 лет $k=700$, для наблюдений менее 100 лет $k=(300: 500)$ В работе под сигналом $f(t)$ понимается кривая изменений исследуемого гелиокосмического фактора климатической переменной. Эти кривые приведены на рис. 1. 
На рис. 2, а в качестве примера представлено изображение временной вейвлетной фазо-частотной функции $\varphi_{f_{1}}(a, b)$ барицентрического движения Солнца $f_{1}(t)$, приведенной на рис. $1, a$ и его вейвлетные фазо-частотная $\varphi_{f_{1}}(a, \bar{b})$ и фазо-временная $\varphi_{f_{1}}(a, b)$ характеристики. Изображение получено при использовании вейвлета 'cgau5' с масштабом $a(1: 1000)$. В работе все вейвлетные преобразования произведены с использованием вейвлета 'cgau5'.
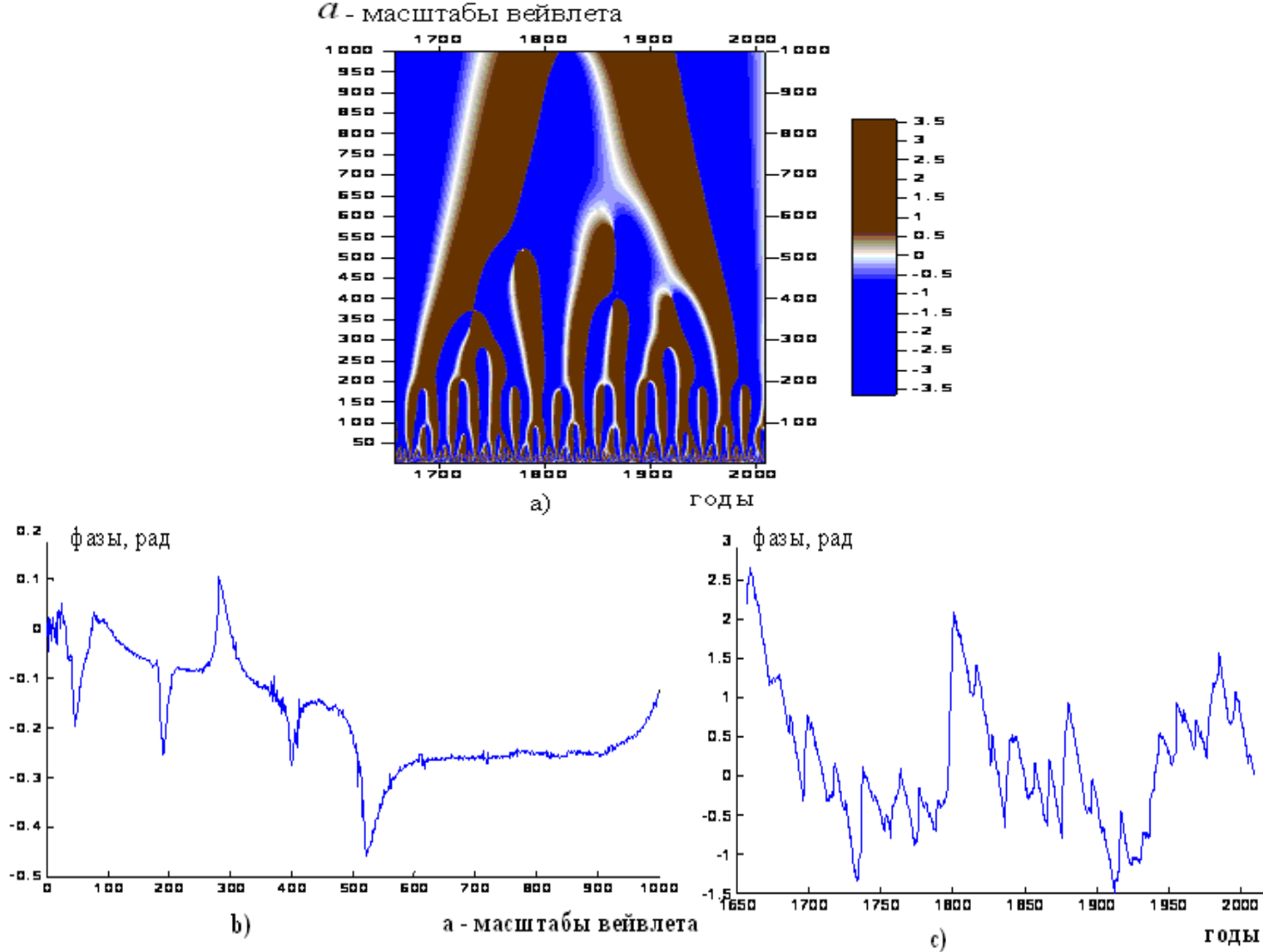

Pис. 2. а) изображение временной вейвлетной фазо-частотной функиии $\varphi_{f_{l}}(a, b)$ изменения расстояния Солнияа до барицентра $f_{l}(t)$, распределенной по масштабу а (ординате) в интервале [1:1000] и времени b (абсииссе) в 1656,5-2009 г2.; b) фазо-частотная характеристика $\varphi_{f_{1}}(a, \bar{b})$; c) фазо-временная характеристика $\varphi_{f_{1}}(a, b)$; фаза сигнала изменяется в интервале $\mp \pi$ радиан. В преобразованиях $\varphi_{f_{1}}(a, \bar{b})$ и $\varphi_{f_{1}}(\bar{a}, b)$ знаки $\bar{a}$ и $\bar{b}$ обозначают операчии усреднений по а и $b$ в преобразовании $\varphi_{f_{1}}(a, b)$

Fig. 2. a) image of the time wavelet phase-frequency function of the change in the distance of the Sun to the barycenter, distributed over the scale (ordinate) in the interval [1:1000] and time (abscissa) in 1656,5-2009; b) phase-frequency response; c) phase-time characteristic; phase of the signal varies in the range $\mp \pi$ of radians. In the formulas $\varphi_{f_{1}}(a, \bar{b})$ and $\varphi_{f_{1}}(a, b)$ signs $\bar{a}$ and $\bar{b}$ denote the averaging operations for and in the conversion $\varphi_{f_{1}}(a, b)$

Изображение функции $\varphi_{f_{1}}(a, b)$ на рис. $2, a$ характеризует ветвление «дерева» низкочастотных фаз сигнала на определенных масштабных уровнях $a$ на высокочастотные составляющие; чередование положительных и отрицательных фаз сигнала формируют периодичность сигнала. Исследуемая функция $f_{1}(t)$ состоит из набора характерных для сигнала гармоник с разными частотами.

На графике фазо-частотной характеристики $\varphi_{f_{1}}(a, \bar{b})$ функции $f_{1}(t)$ отображается изменение фазы сигнала при изменении масштаба $a$ вейвлета от меньшего к большему (от большей частоты сигнала к менышей). На графике выделяются особые точки с острыми пиками, направленными вниз и вверх, кото- рым соответствуют определенные вейвлетные масштабы $a$; при этих значениях вейвлетных масштабов $a$ происходят бифуркации частот сигнала, а иногда и утроение частоты, наблюдаемые на рис. 2, $a$. Наличие бифуркаций на вейвлетных фазо-частотных функциях $\varphi_{f_{1}}(a, b)$ сигналов $f_{i}(t)$ является признаком нелинейности процесса изменения исследуемой переменной, признаком нелинейности колебательной системы $[26,30]$. Как известно, выходами таких систем могут быть квазипериодические, субгармонические и хаотические колебания. Из рис. 2, $b$ следует, что бифуркации частот сигнала $f_{1}(t)$ происходили при значениях $a \approx 1000,524,401,282,191$ и 47. Изменения функции $\varphi_{f_{1}}(a, \bar{b})$ между особыми точками также характери- 
зуют особенности структуры функции $f_{1}(t)$, изменения ее частоты в зависимости от вейвлетного масштаба. Функция $\varphi_{f_{1}}(a, b)$ является отличительным, индивидуальным (уникальным) признаком сигнала $f_{1}(t)$, его «отпечатком пальца». График функции $\varphi_{f_{1}}(a, b)$ на рис. 2, $c$ характеризует изменение усредненной по вейвлетным масштабам мгновенных фаз сигнала $f_{1}(t)$ в наблюдаемом интервале времени (фазо-временная характеристика). Заметим, что такие особенности изменений сигнала не могут быть получены при Фурье-анализе сигнала.

Фазо-временная характеристика преобразования $\varphi_{f}(a, b)$ вычисляется как $\varphi_{f}(a, b)=-\varphi_{f}(a, b)$, знак минус перед преобразованием необходим для согласования изменений фазо-временной характеристики $\varphi_{f}(a, b)$ с изменениями исходной функции $f(t)$. Можно утверждать, что функции $\varphi_{f}(a, b), \varphi_{f}(a, b)$ и $\varphi_{f}(a, \bar{b})$ являются основными отличительными признаками любых исследуемых функций $f(t)$ и они в работе используются для установления причинноследственных связей между переменными, определяющими климат на Земле.

\section{Применение вейвлетных фазо-частотных и фазо-временных характеристик для оценивания согласованности (коррелированности) изменений климатических переменных, полученных на станции «Восток» в Антарктиде}

В продолжительных временных интервалах наблюдений нестационарные сигналы $f_{i}(t), i=1, \ldots, k$ могут подвергаться существенным изменениям как по частотному, так и по фазовому составам в результате действия разных факторов. В этой связи вейвлетные характеристики $\varphi_{f}(a, \bar{b})$ и $\varphi_{f}(\bar{a}, b)$ могут быть использованы для оценивания коррелированности сравниваемых функций $f_{i}(t)$, $i=1, \ldots, l ; l-$ число сравниваемых функций.

Таблица 1. Коэффициенты линейных корреляций между изменениями орбитально-климатической диаграммы (ОКД) $f_{\text {OKD }}(t)$ [31], изменениями температуры $f_{\text {Темр }}(t)$ и двуокиси углерода $f_{\mathrm{CO}_{2}}(t)$ на планете в интервале времени от 800 тыс. лет в прошлом до современности [32]

Table 1. Linear correlation coefficients between changes in the orbital-climatic diagram (OKD) [31], changes in temperature and carbon dioxide on the planet in the time interval from 800 thousand years in the past to the present [32]

\begin{tabular}{|c|c|c|c|c|c|c|c|c|c|}
\hline \multirow{2}{*}{$\begin{array}{c}\text { условия } \\
\text { terms }\end{array}$} & \multicolumn{3}{|c|}{$\begin{array}{c}\varphi_{f}(a, \bar{b}) \text { - фазо-частотные } \\
\text { характеристики, } a=1: 1500 \\
\text { phase-frequency characteristics }\end{array}$} & \multicolumn{3}{|c|}{$\begin{array}{c}\varphi_{f}(a, b)-\text { фазо-временные } \\
\text { характеристики, } a=1: 1500 \\
\text { phase-time characteristics }\end{array}$} & \multicolumn{3}{|c|}{$\begin{array}{c}\text { Исходные временные } \\
\text { функции } f(t) \\
\text { Initial time functions } f(t)\end{array}$} \\
\hline & $f_{O K D}(t)$ & $f_{\text {Tenp }}(t)$ & $f_{\mathrm{CO}_{2}}(t)$ & $f_{O K D}(t)$ & $f_{\text {Tемр }}(t)$ & $f_{\mathrm{CO}_{2}}(t)$ & $f_{O K D}(t)$ & $f_{\text {Teмp }}(t)$ & $f_{\mathrm{CO}_{2}}(t)$ \\
\hline$f_{O K D}(t)$ & 1,00 & & & 1,00 & & & 1,00 & & \\
\hline$f_{\text {Temp }}(t)$ & $-0,58$ & 1,00 & & 0,42 & 1,00 & & 0,46 & 1,00 & \\
\hline$f_{\mathrm{CO}_{2}}(t)$ & 0,67 & $-0,07$ & 1,00 & 0,05 & 0,46 & 1,00 & 0,29 & 0,85 & 1,00 \\
\hline
\end{tabular}

Примечание. Коэффициенты корреляций $r>|0,065|$ значимы с вероятностью 0,95 по критерию Стьюдента [33].

Note. Correlation coefficients $r>|0,065|$ are significant with a probability of 0,95 according to Student's criterion [33].

Корреляции вычислены в вариантах с использованием вейвлетных преобразований $\varphi_{f}(a, \bar{b}), \varphi_{f}(a, b)$ и без них. Кривая изменений $f_{\text {OKD }}(t)$ является характеристикой изменений климата на Земле, инсоляцией, обусловленной изменениями элементов орбиты Земли (эксцентриситета, угла наклона и климатической прецессии со знаком «минус» в пропорции 1:0,7:0,55) [31]. Эффективный метод прогнозирования кривой ОКД(t) с использованием многомасштабных вейвлетов изложен в работе [34].

При корреляционном анализе изменчивостей переменных с использованием вейвлетных преобразований $\varphi_{f}(a, \bar{b})$ и $\varphi_{f}(\bar{a}, b)$ необходимо учитывать следующие обстоятельства:

1) при использовании преобразования $\varphi_{f}(a, \bar{b})$ коэффициенты корреляций характеризуют меру присутствия фазо-частотной характеристики $\varphi_{f_{i}}(a, \bar{b})$ в $\varphi_{f_{j}}(a, \bar{b})$, меру причинной обусловленности переменных по аналогии с принципом причинности Грэнджеpa [35]. Модули коэффициентов корреляций являются мерами согласованности характеристик по частотам, а их знаки - согласованности по фазам; 2) при исполь- зовании преобразования $\varphi_{f}(\bar{a}, b)$ коэффициенты корреляций описывают меру присутствия фазо-временной характеристики $\varphi_{f_{i}}(a, b)$ в $\varphi_{f_{j}}(a, b)$, согласованность изменений переменных по фазам и времени. Эти коэффициенты корреляций отражают меру влияния на изменчивость переменной $f_{i}(t)$ других климатических факторов в наблюдаемом интервале времени. В табл. 1 и на рис. $3, a, b$ отражаются закономерности взаимосвязанных изменений переменных в продолжительном интервале времени в трех вариантах: фазо-частотной, фазо-временной и временной, в присутствии других воздействующих факторов. Как видно, между ними есть существенные отличия, характеризующие структуры пространств, в которых они изменяются. Например, изменения приземной температуры $f_{\text {Teмp }}(t)$ и двуокиси углерода $f_{\mathrm{CO}_{2}}(t)$ происходят не только под влиянием инсоляции $f_{\text {OKD }}(t)$, но и под воздействием температуры мирового океана $f_{19}(t)$, вулканических извержений $f_{11}(t)$ и многих других факторов. Из табл. 1 также видно, что вычисления корреляций между переменными только во временной области, знания только частотного состава переменных, недостаточно для установления взаимо- 
влияний между переменными. Другим свойством функций $\varphi_{f}(a, b)$ и $\varphi_{f}(a, b)$ является то, что плотности распределений этих функций дают представление о числе основных факторов, влияющих на изменения функций $f_{i}(t)$ и их спектральных составах. На графиках $c, d$ и $e$ рис. 3 приведены кривые плотностей распределений вейвлетных фазо-временных характеристик: $\varphi_{\text {OKD }}(\bar{a}, b)$, $\varphi_{\text {Tетр }}(a, b)$ и $\varphi_{\mathrm{CO}_{2}}(a, b)$, которые состоят из смесей плотностей распределений нескольких существенных факторов [36], каждый из них формируется влияниями нескольких переменных, изменяющихся разными периодичностями.
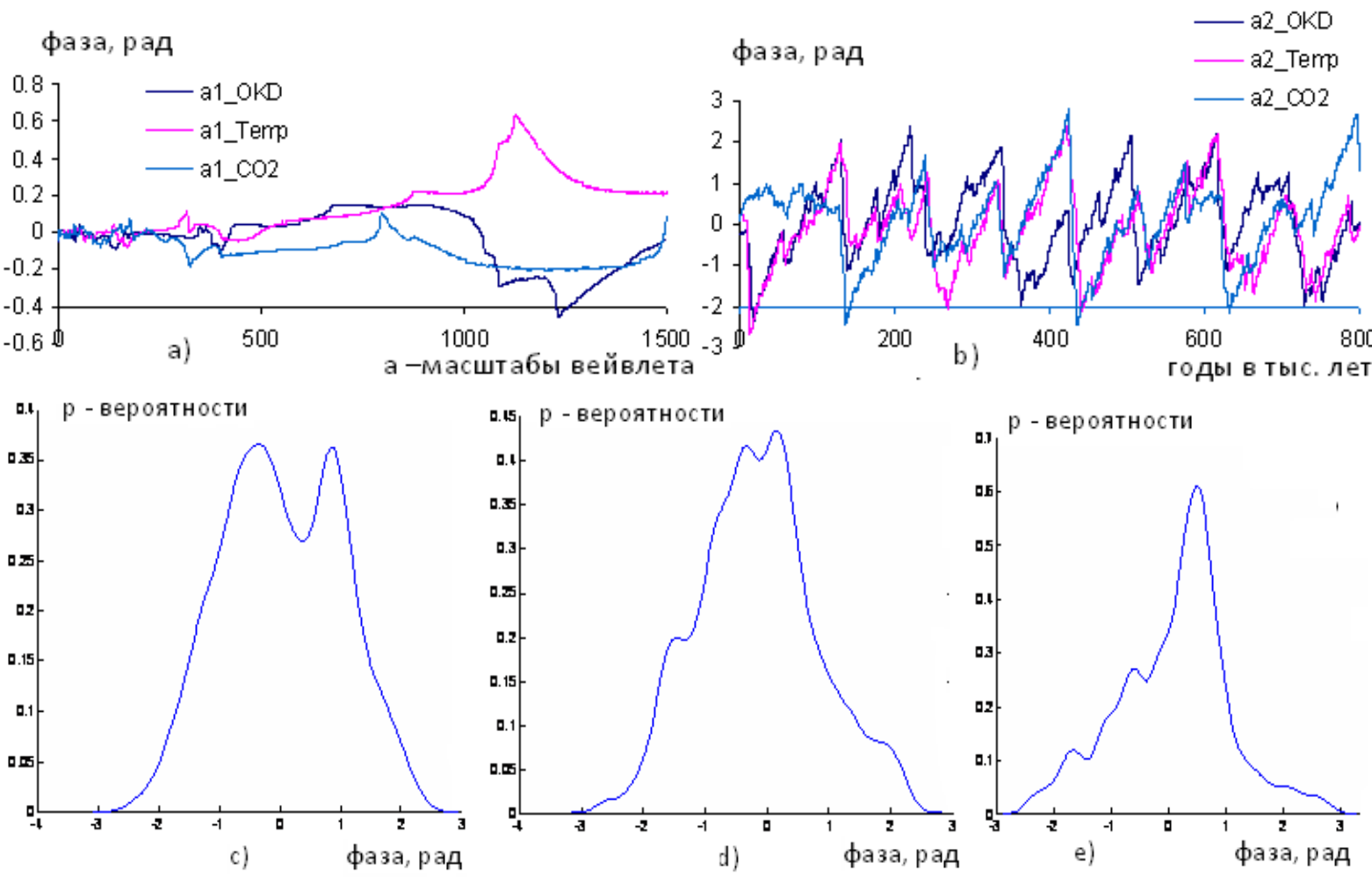

Pис. 3. а) фазо-частотные $\varphi_{f}(a, \bar{b})$, b) фазо-временные $\varphi_{f}(\bar{a}, b)$ характеристики и плотности распределений вейвлетных фазо-временных характеристик орбитально-климатической диаграммы (ОКД) и климатических переменных по данным исследований кернов Антарктического льда в интервале времени 800 тыс. лет в прошлом [31]: с) ОКД(t), построенной в пропориии (1:0,7:0,55); d) приземной температуры Темр $(t) ; e)$ двуокиси углерода $\mathrm{CO}_{2}(t)$ в Антарктиде. Фазы переменных изменяются в интервале 干л по абсииссе. Вейвлетные преобразования выполнены при масштабах вейвлета а(1:1500)

Fig. 3. a) phase-frequency, b) phase-time characteristics and densities of distributions of wavelet phase-time characteristics of the orbital-climatic diagram (OKD) and climate variables according to studies of Antarctic ice cores in the time interval of 800 thousand years in the past [31]: c) OKD (t), built in proportion (1:0,7:0,55); d) surface temperature; e) carbon dioxide in Antarctica. The phases of the variables vary in the interval $\mp \pi$ on the abscissa. Wavelet transforms performed at wavelet scale a(1:1500)

\section{Вычисление частотного состава (периодичности) функций $y(t)=f(t)$}

Изображение временной вейвлетной фазочастотной функции исследуемой функции $\varphi_{f}(a, b)$, представленное на рис. 2, a, подчеркивает периодичность функции $f(t)$ при заданных масштабах $a$ вейвлета, ее спектральный состав и подсказывает метод оценивания этой периодичности. Вычисление периодичности функции $\varphi_{f}(a, b)$ состоит из последовательности шагов:

а) для заданного значения масштаба $а$ вычисляются значения функции $\varphi_{f_{1}}(a, b)$ на всем интервале изменений $b$; на рис. 4 приведен график функции $\varphi_{f_{1}}(a, b)$, вычисленный при $a=150$, временной параметр $b$ изменяется в интервале 1656,5-2009 гг. с шагом $\Delta b=0,1$ год. Как видно из рисунка, график разреза функции циклический, почти периодический.

б) оцениваются временные координаты $\hat{T}_{i}, i=1: k$ максимумов или минимумов полученного графика функции $\varphi_{f_{1}}(a, b)$, где $k$ - количество экстремумов функции;

в) вычисляется среднее значение $T_{a}=\operatorname{mean}\left(T_{i}, i=1: k\right)$ периодичности функции $\varphi_{f_{1}}(a, b)$ для заданного значения $a$ и его стандартное отклонение $\sigma_{a}=\operatorname{std}\left(T_{i}, i=1: k\right)$ относительно среднего периода $T_{a}$, если $k \geq 3$.

Если график функции $\varphi_{f_{1}}(a, b)$ окажется зашумленным, то с целью более точного оценивания координат $T_{i}$ экстремумов может быть использовано ее сглаживание с использованием стандартных процедур; 
г) задавая значения характерного масштаба $a$ от большего к меньшему вычисляется весь спектральный состав исследуемой функции $f(t)$ с оцениванием средних значений периодичностей $\hat{T}_{f}$ и их стандартных отклонений $\sigma_{T_{f}}$

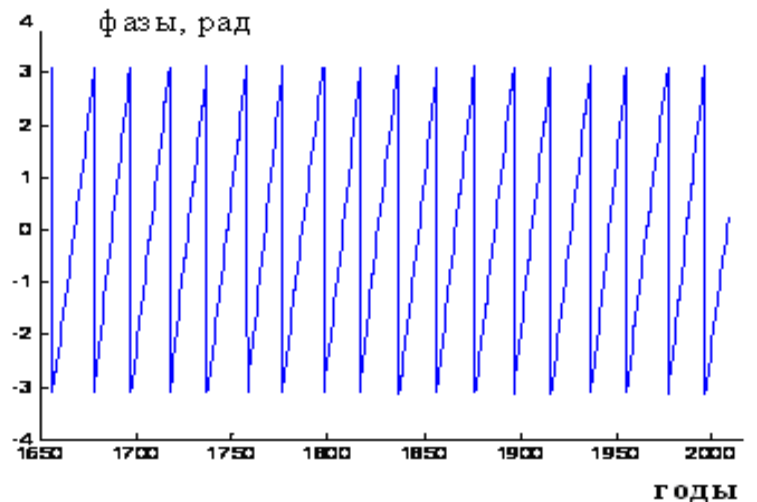

Рис. 4. График функиии $\varphi_{f_{I}}(a, b)$ в радианах в интервале $\mp \pi$, вычисленный при $а=150$ в интервале времени $1656,5-2009$ z2.

Fig. 4. Graphic of the function in radians in the interval $\mp \pi$ calculated for $a=150$ in 1656,5-2009
В изменениях приведенных выше факторов (функций) существует глубокая связь. Изменения каждой из этих функций в той или мере обусловлены изменениями других факторов. Для установления причинно-следственных связей, согласованности в изменениях переменных вычисляются коэффициенты корреляций между вейвлетными фазо-частотными характеристиками переменных $f_{1}-f_{24}$; графики переменных $f_{21}-f_{24}$ приведены во второй части статьи.

Совместный корреляционный анализ

гелиокосмических и климатических переменных по наблюдениям в 1955-2006 гг.

В табл. 2 наблюдается высокая согласованность изменений пар $\left(f_{1}, f_{5}\right),\left(f_{1}, f_{11}\right),\left(f_{5}, f_{11}\right),\left(f_{6}, f_{10}\right),\left(f_{1}, f_{11}\right)$, $\left(f_{6,} f_{24}\right),\left(f_{10}, f_{11}\right),\left(f_{10}, f_{16}\right),\left(f_{11}, f_{16}\right)$ и других пар, характеризующих высокую согласованность изменений гелиокосмических переменных $f_{1}, f_{2}$, солнечной активности $f_{5}$, земных климатических переменных $f_{6}, f_{7}, f_{10}$, $f_{11}-f_{14}$, характеризующих тектонические процессы на Земле, переменных $f_{15}-f_{23}$, определяющих температурный режим на Земле, и переменную $f_{24}$, характеризующую электромагнитную связь между изменениями солнечных и земных переменных.

Таблица 2. Коэффициенты влияния гелиокосмических переменных, солнечной активности и вулканической деятельности Земли на изменения приземной температуры, климатические изменения, происходящие на поверхности океанов в 1955-2006 г2., вычисленные с вейвлетныли преобразованиями $\varphi_{f_{i}}(a(1: 700), \bar{b})$, $i=1, \ldots, 24$

Table 2. Coefficients of the effect of solar-cosmic variables, solar activity and the Earth's volcanic activity on changes in surface temperature, climatic changes occurring on the surface of the oceans in 1955-2006, calculated with wavelet transformations $\varphi_{f_{i}}(a(1: 700), \bar{b}), i=1, \ldots, 24$

\begin{tabular}{|c|c|c|c|c|c|c|c|c|c|c|c|c|c|c|c|c|}
\hline$f$ & $f_{1}$ & $f_{2}$ & $f_{5}$ & $f_{6}$ & $f_{10}$ & $f_{11}$ & $f_{15}$ & $f_{16}$ & $f_{17}$ & $f_{18}$ & $f_{19}$ & $f_{20}$ & $f_{21}$ & $f_{22}$ & $f_{23}$ & $f_{24}$ \\
\hline$f_{1}$ & 1 & & & & & & & & & & & & & & & \\
\hline$f_{2}$ & 0,30 & 1 & & & & & & & & & & & & & & \\
\hline$f_{5}$ & 0,94 & 0,30 & 1 & & & & & & & & & & & & & \\
\hline$f_{6}$ & $-0,73$ & $-0,75$ & $-0,74$ & 1 & & & & & & & & & & & & \\
\hline$f_{10}$ & 0,64 & 0,26 & 0,79 & $-0,60$ & 1 & & & & & & & & & & & \\
\hline$f_{11}$ & 0,84 & 0,23 & 0,94 & $-0,67$ & 0,88 & 1 & & & & & & & & & & \\
\hline$f_{15}$ & 0,32 & 0,36 & 0,39 & $-0,49$ & 0,32 & 0,30 & 1 & & & & & & & & & \\
\hline$f_{16}$ & 0,78 & 0,15 & 0,90 & $-0,65$ & 0,86 & 0,94 & 0,34 & 1 & & & & & & & & \\
\hline$f_{17}$ & 0,44 & 0,54 & 0,42 & $-0,59$ & 0,33 & 0,33 & 0,32 & 0,34 & 1 & & & & & & & \\
\hline$f_{18}$ & 0,46 & 0,28 & 0,52 & $-0,57$ & 0,38 & 0,44 & 0,74 & 0,56 & 0,19 & 1 & & & & & & \\
\hline$f_{19}$ & $-0,11$ & 0,26 & $-0,07$ & $-0,05$ & $-0,06$ & $-0,09$ & 0,36 & $-0,15$ & $-0,47$ & 0,29 & 1 & & & & & \\
\hline$f_{20}$ & 0,19 & 0,30 & 0,17 & $-0,30$ & 0,07 & 0,07 & 0,56 & 0,09 & 0,21 & 0,35 & 0,40 & 1 & & & & \\
\hline$f_{21}$ & $-0,05$ & 0,03 & $-0,08$ & 0,06 & $-0,13$ & $-0,15$ & 0,30 & $-0,14$ & 0,03 & 0,03 & 0,29 & 0,84 & 1 & & & \\
\hline$f_{22}$ & 0,18 & 0,17 & 0,22 & $-0,29$ & 0,23 & 0,22 & 0,72 & 0,28 & $-0,14$ & 0,73 & 0,61 & 0,42 & 0,19 & 1 & & \\
\hline$f_{23}$ & $-0,08$ & $-0,59$ & $-0,10$ & 0,39 & $-0,29$ & $-0,16$ & 0,11 & $-0,10$ & $-0,33$ & 0,03 & 0,14 & 0,38 & 0,55 & 0,23 & 1 & \\
\hline$f_{24}$ & 0,64 & 0,80 & 0,60 & $-0,88$ & 0,36 & 0,49 & 0,47 & 0,46 & 0,62 & 0,55 & 0,01 & 0,21 & $-0,15$ & 0,27 & $-0,51$ & 1 \\
\hline
\end{tabular}

Примечание: вычисленные коэффичиенты коррелячии по критерию Стьюдента значимы при аbs(r)>0,1 с вероятностью $p=0,95$.

Note: the calculated correlation coefficients $a b s(r)>0,1$ by the Student criterion are significant at probability $p=0,95$.

Как в наборе периодов циклических движений Солнца, так и в наборе частот вариаций солнечной активности присутствуют такие периоды в годах, как $4,85(1,4), 10,65(1,4), 12,95(3,86), 22(9,6), 44(7,45)$, $66,9(13,2), 112(12)$ (в скобках указаны стандартные отклонения $\sigma$ от средних). В частотных составах изменений расстояния центра масс Солнца от барицентра $f_{1}(t)$, отклонений угловой скорости вращения Земли от эталонной $f_{7}(t)$ в интервале времени с 1656 по
2008 гг. и в вариациях солнечной активности в интервале времени 1610-2010 гг., наблюдаются частоты с периодами $\approx 178, \approx 36$ и $\approx 11,5$ лет. В асимметрии движения Солнца присутствуют гармоники с периодами 46,6 (3,4), 35,87 (2,3), 19,6 (2,1), 12,8 (4,6), соответствующие синодическим периодам Сатурна/Урана, Сатурна/Нептуна, Сатурна/Юпитера и Юпитера/Нептуна соответственно. Эти периоды являются целыми кратными 179-летнего цикла Жозе [37]. Эти и 
другие частоты, наблюдаемые в движениях Солнца, хорошо коррелируются с периодами солнечной активности. Такие выводы получены и в работах [3846]. Известна согласованность периодических движений планет солнечной системы и их гравитационное взаимодействие [47].

Все движения Солнца отражаются на движении Земли и на ее глубинных процессах, в том числе и в виде случайных составляющих [30]. Эти процессы проявляются в виде циклических изменений скорости вращения Земли (строка и столбец $f_{6}$ в табл. 2) с высокими отрицательными коэффициентами корреляции. Отрицательный знак корреляции характеризует неоднородность строения Земли: любые изменения в угловом импульсе жидкой части земной системы, вызванные движением Солнца и его планет относительно Земли, должны быть скомпенсированы равным и противоположным по знаку изменением в угловом импульсе твердой Земли [48].

Таблица 2 является обобщенной характеристикой связей между нелинейными взаимосвязанными колебательными контурами Солнечно-Земной системы. В этой системе движения планет солнечной системы приводят к изменениям солнечной активности $f_{5}$ и вулканических извержений $f_{11}$, других тектонических движений на Земле. В свою очередь, вулканические извержения тесно согласуются с изменениями $\mathrm{CO}_{2} f_{16}$ и изменениями скорости вращения Земли $f_{6}$ и продолжительности земных суток LOD $f_{10}$.

Из табл. 2 коэффициентов корреляций изменений климатических факторов следует, что:

- солнечная энергия, получаемая Землей, перераспределяется в тепловую энергию воздуха суши, тепловую энергию воздуха на поверхности Мирового океана и тепловую энергию толщи воды океана. В этом процессе значительное место занимает вулканическая деятельность Земли, при которой из недр в атмосферу Земли выбрасывается огромная масса парниковых газов, в том числе и углекислый газ $\mathrm{CO}_{2}$;

- изменение приземной температуры (столбец $f_{15}$ ) в значительной мере согласовано с изменениями $\mathrm{CO}_{2}$ в атмосфере $f_{16}(r=0,34)$, Тихоокеанского декадного колебания (PDO) $f_{18}$, глобальной температуры воздуха над сушей $f_{19}$, теплосодержания океана в ее толще $f_{21}$, аномальной температуры океана в зоне от $60^{\circ} S$ до $60^{\circ} N f_{22}$, вариаций Южного колебания/Эль-Ниньо $f_{23}$ с коэффициентами от 0,11 до 0,74. Источником тепловой энергии атмосферного воздуха (строка $f_{15}$ ) является солнечная активность $f_{5}$ с коэффициентом 0,39; на ее изменчивость влияют изменчивость длительности суток $f_{10}$ и вулканическая деятельность Земли $f_{11}$, а также скорость вращения Земли $f_{6}$;

- температурная неоднородность океана и атмосферы Земли приводят к движениям водных и воздушных масс в огромных пространственных масштабах, устанавливаются самоорганизующиеся колебательные процессы Североатлантической, Тихоокеанской декадной и Южной/Эль-Ниньо ко- лебаний набором периодичностей. Эти периодичности согласованы с периодичностями асимметричных движений Солнца относительно его барицентра;

- вариации индекса Южных колебаний/Эль-Ниньо $f_{23}$ синхронизированы с аномалиями роста температуры $f_{22}$ поверхности океана в зоне от $60^{\circ} S$ до $60^{\circ} \mathrm{N}$, теплосодержанием 700 метрового слоя океана $f_{21}$, глобальной температурой воздуха на поверхности океана $f_{20}$, глобальной температурой воздуха в районе суши $f_{19}$, изменениями скорости вращения Земли $f_{6}$ и изменениями приземной температуры $f_{15}$; изменение переменной $f_{23}$ находится в противофазе с изменениями длительности суток LOD $f_{10}$, извержений вулканов $f_{11}$, изменениями концентраций $\mathrm{CO}_{2} f_{16}$ и индекса Североатлантического колебания $f_{17}$;

- аномалии роста теплосодержания океана $f_{21}$ в толще воды 0-700 м синхронизированы ростом глобальной температуры приземной температуры $f_{15}$, аномалиями роста температуры воздуха на поверхности Мирового океана $f_{20}$ и аномалиями роста температуры воздуха над сушей $f_{19}$; находятся в противофазе с изменениями длительности суток $f_{10}$, вулканических извержений $f_{11}$ и изменений $\mathrm{CO}_{2}$ в атмосфере;

- в строке $f_{24}$ табл. 2 отражены меры согласованности изменений числа магнитных бурь с изменениями солнечной активности $f_{5}$, вулканической деятельности Земли $f_{11}$, концентрации в атмосфере углекислого газа $f_{16}$ и колебательных процессов, происходящих в атмосфере Земли и в океане, факторов $f_{17}-f_{23}$. Интенсивность магнитных бурь тесно согласована с активностью Солнца, вулканическими извержениями на Земле, изменениями приземной температуры и $\mathrm{CO}_{2}$ в атмосфере, индексов Североатлантического и Тихоокеанского декадных колебаний. Значительно согласованы изменения магнитных бурь с Южными колебаниями/ Эль-Ниньо $f_{23}$ и изменениями скорости вращения Земли $f_{6}$ с отрицательными знаками, $r=-0,51$ и $r=-$ 0,88 соответственно; знак коэффициентов корреляций между переменными зависит от продолжительности наблюдений и выбранного для анализа масштаба $a$ вейвлета. В работе [14] отмечена коррелированность изменений Североатлантического и Тихоокеанского декадного колебаний с изменениями скорости вращения Земли и возможная связь этой зависимости с асимметриями в движении Солнца вокруг центра масс солнечной системы.

Из табл. 2 коэффициентов линейных корреляций между вейвлетными фазо-частотными характеристиками $\varphi_{f_{i}}(a, \bar{b})$ сравниваемых факторов в одинаковых интервалах времени, определяющих климат на Земле, следует, что:

- вулканическая деятельность на Земле индуцируется асимметричным движением Солнца относительно барицентра, формируемого планетами Солнечной системы, при этом циклические про- 
цессы на Солнце активизируются приливными, гравитационными силами, формируемыми планетами-гигантами солнечной системы (Юпитером, Сатурном, Ураном, Нептуном);

- возмущенные движения Солнца гравитационными силами передаются на процессы, происходящие в атмосфере Земли и в недрах неоднородной Земли, возбуждая в ней вулканические, геомагнитные и другие процессы. Вулканические, тектонические процессы на Земле активизируются также приливными силами Луны;

- имеется высокая согласованность изменений концентрации $\mathrm{CO}_{2}$ в атмосфере Земли с изменениями интенсивности вулканических извержений с коэффициентами $r=0,88-0,94$ в разных временных интервалах наблюдений.

В природе все процессы происходят в циклическом режиме и согласованы с асимметричными периодическими движениями Солнца относительно барицентра, периодическими изменениями солнечной активности и вулканической деятельности Земли. Причем периоды изменений процессов, происходящих в природе, отличаются друг от друга. По этой причине представляет интерес сравнительное исследование изменений этих процессов в одних и тех же временных интервалах, позволяющих предсказать будущее этих изменений с заданной периодичностью (при заданных значениях масштаба $a$ вейвлета) с использованием вейвлетных фазо-временных характеристик $\varphi_{f_{i}}(\bar{a}, b)$. Результаты этих и других исследований представлены во второй части работы.

\section{Выводы}

1. Разработан метод, позволяющий извлечь из временных рядов наблюдений $f(t)$ наиболее существенные характеристики, отличающие их от других временных рядов. Такими характеристиками любого сигнала являются: временная вейвлетная фазочастотная функция $\varphi_{f}(a, b)$ и ее вейвлетные фазочастотная $\varphi_{f}(a, \bar{b})$ и фазо-временная $\varphi_{f}(\bar{a}, b)$ характеристики, позволяющие идентифицировать климатические изменения на Земле.

\section{СПИСОК ЛИТЕРАТУРЫ}

1. Сигналы и последствия изменений климата сохраняются в 2018 году // Пресс-релиз Всемирной метеорологической организации. Номер документа 29112018. Дата публикации 29 ноября 2018 г. URL: https://public.wmo.int/ru/media/прессрелизы/сигналы (дата обращения 20.05.2019).

2. Глобальное потепление на $1,5{ }^{\circ} \mathrm{C} / /$ Доклад межправительственной группы экспертов по изменению климата (МГЭИК). Опубликован 8 октября 2018 г. URL: www.meteorf.ru/upload/iblock/f5e/Izmenenie_klimata_N75OctNo v_2018.pdf (дата обращения 20.05.2019).

3. Итоги конференции по изменению климата в Катовице СОП24. - Польша, 2018. Дата публикации 15.12.2018. URL: http://ecounion.kz/?p=716 (дата обращения 20.05.2019).

4. Доклад об особенностях климата на территории Российской Федерации за 2018 год. - M., 2019. - 79 с. URL: http://meteo.ru/events/102-raznoe/754-doklad-ob-osobennostyakhklimata-na-territorii-rossijskoj-federatsii-za-2018-god (дата обращения 20.05.2019).
2. Вейвлетные характеристики $\varphi_{f}(a, \bar{b})$ и $\varphi_{f}(\bar{a}, b)$ являются индивидуальными для каждой функции $f(t)$ и зависят от влияний других климатических факторов системы, изменяющихся во времени, выбранного для анализа масштаба $a$ вейвлета.

3. Вычислением коэффициентов корреляций между характеристиками $\varphi_{f_{i}}(a, \bar{b})$ сравниваемых переменных $i=1, \ldots, 24$, изменяющихся в 1955-2006 гг., установлено, что изменчивости солнечной активности $f_{5}$, вулканических извержений $f_{11}$, скорости вращения Земли $f_{6}$, продолжительности земных суток $f_{10}$ сильно согласованы с изменениями расстояния Солнца относительно барицентра $f_{1}$.

4. Изменение концентрации двуокиси углерода $\mathrm{CO}_{2}$ $f_{16}$ в атмосфере сильно согласовано с изменениями вулканических извержений $f_{11}$, обусловленными изменениями $f_{1}$.

5. Изменения приземной температуры $f_{15}$ согласованы с изменениями $f_{5}, f_{16}$ и изменениями теплосодержания океана $f_{21}$, температуры океана в экваториальной зоне $f_{22}$, температуры воздуха на поверхности мирового океана $f_{20}$, температурных изменений Тихоокеанских колебаний $f_{18}$, изменений индекса $\mathrm{Ce}$ вероатлантических колебаний $f_{17}$ и Эль-Ниньо $f_{23}$.

6. Изменения магнитных бурь $f_{24}$ согласованы с изменениями многих переменных $f_{1}-f_{18}$ и $f_{23}$.

7. В матрице табл. 2 проявляются многоуровневые синхронизованные изменения гелиокосмических и климатических переменных $f_{12}-f_{24}$, обусловленные изменениями переменной $f_{1}$ - движением центра масс (ц.м) Солнца относительно ц.м. солнечной системы в фазо-частотной области.

8. Временная вейвлетная функция $\varphi_{f}(a, b)$ и ее характеристики $\varphi_{f}(a, \bar{b}), \varphi_{f}(\bar{a}, b)$ весьма информативны и удобны для наглядного графического представления, сравнения исследуемых функций, анализа, интерпретации результатов исследований объектов произвольной природы; информативные преимущества вновь введенных функций используются для уточнения и развития роли изменений гелиокосмических переменных в изменениях природной среды во второй части работы.

5. Доклад о климатических рисках на территории Российской Федерации // Федеральная служба по гидрометеорологии и мониторингу окружающей среды (РОСГИДРОМЕТ). Климатический центр. - СПб., $2017 . \quad$ - 106 с. URL: http://cc.voeikovmgo.ru/images/dokumenty/2017/riski.pdf (дата обращения 20.05.2019)

6. Rosenzweig R. Global climate change policy and carbon markets. - London: Springer, 2016. - 322 p.

7. Carbon markets for greenhouse gas emission reduction in a warming world an evaluation of the world bank group's support to carbon finance. International Bank for Reconstruction and Development / The World Bank. Washington, 2018. - 227 p.

8. Shiferaw B., Store A. Builing the next generation of carbon markets for climate change mitigation // Indpendent Evaluation Group (IEG). World Bank Group. 2019

9. Wang S., Lee W., Son Y. Low carbon development pathways in Indian agriculture // Change Adaptation Socio-Ecological Systems. - 2017. - V. 3. - P. 18-26. 
10. Gordon E., Lieberman B. Climate change in human history: prehistory to the present. - London: Bloomsbury Academic \& Professional, 2018. - 244 p.

11. Современные глобальные изменения природной среды. В 2-х т. Т. 1 / под ред. Клиге Р.К., Лукьяновой С.А. - М.: Научный мир, 2006. -696 с.

12. Современные глобальные изменения природной среды. В 2-х т Т. 2 / под ред. Клиге Р.К., Лукьяновой С.А. - М.: Научный мир, 2006. $-776 \mathrm{c.}$

13. Современные глобальные изменения природной среды. Т. 3 Факторы глобальных изменений / под ред. Клиге Р.К., Хлыстова А.И. - М.: Научный мир, 2012. - 444 с.

14. Хлыстов А.И., Клиге Р.К., Сидоренков Н.С. Резонанс третьего рода в Солнечной системе // Современные глобальные изменения природной среды. Т. 3. Факторы глобальных изменений. - М.: Научный мир, 2012. - С.147-160.

15. Вильсон Ян Р.Г. Изменение во вращении Земли относительно барицентра и климатический эффект // Современные глобальные изменения природной среды. Т. 3. Факторы глобальных изменений. - М.: Научный мир, 2012. - С. 78-102.

16. Хлыстов А.И., Долганов В.П., Доможилова Л.М. Барицентрическое движение Солнца и его следствия для Солнечной системы // Современные глобальные изменения природной среды. Т. 3. Факторы глобальных изменений. - М.: Научный мир 2012. - С. 62-77.

17. Сидоренков Н.С. Нестабильности вращения Земли и глобальные изменения природных процессов // Современные глобальные изменения природной среды. Т. 2. - М.: Научный мир, 2006. - С. 737-747.

18. Касимов Н.С., Клиге Р.К. Введение. Проблемы глобальных изменений // Современные глобальные изменения природной среды. Т. 3. Факторы глобальных изменений. - М.: Научный мир, 2012. - С. 19-34.

19. Милецкий Е.В., Наговицын Ю.А. Магнитные поля солнечных пятен в 21-22 циклах солнечной активности // Известия Государственной Астрономической Обсерватории. - 2000. T. 215 . - C. $259-270$

20. Volkano eruptions of 2014 and all eruptions since 1600 to present // The Big Wobble. 18 June 2016. URL: http://www.thebigwobble. org/2016/06/volcano-eruptions-of-2014-and-all.htmll (дата обращения 20.05.2019).

21. Хаин В.Е., Халилов Э.Н. Цикличность геодинамических процессов: ее возможная природа. - М.: Научный мир, 2009. $520 \mathrm{c}$.

22. Рост природных катаклизмов за последнее столетие по данным Центра исследования развития катастроф (CRED). URL: https://www:liveinternet.ru/users/famar/post367117884 (дата обращения 20.05.2019)

23. Гладильщикова А.А., Семенов С.М. Межправительственная группа экспертов по изменению климата (МГЭИК): цикл шестого оценочного доклада // Фундаментальная и прикладная климатология. - 2017. - Т. 2. - С. 13-25.

24. Hurrell J. National Center for Atmospheric Research (NCAR) 2007. URL: http://www.cgd.ucar.edu/cas/jhurrel/Data/ naodjfmindex.asc (дата обращения: 20.05.2019).

25. Алексеев В.И. Вейвлетный фазо-чстотный анализ климатических переменных по данным глубокого бурения ледниковых скважин в Антарктиде // Динамика окружающей среды и глобальные изменения климата. - 2013. - Т. 4. - № 2. - С. 9-26.

26. Алексеев В.И. Вейвлет-анализ динамики изменений явления Эль-Ниньо и его прогнозирование // Вестник Югорского государственного университета. - 2018. - Т. 3 (50). - С. 75-87.
27. Блаттер К. Вейвлет-анализ. Основы теории. - М.: ТЕХНОСФЕРА, 2004. - $280 \mathrm{c}$.

28. Дьяконов В.П. Вейвлеты. От теории к практике. - М.: СОЛОН-Пресс, 2004. - 400 с.

29. Малла С. Вейвлеты в обработке сигналов / Пер. с англ. - М: Мир, 2005. - 671 с

30. Moon F.C. Chaotic vibration. An introduction for applied scientists and engineers. - Ithaca, New York: Theoretical and Applied Mechanics Cornel University, 1990. - 309 p.

31. Большаков В.А. Новая концепция орбитальной теории палеоклимата. - М.: МГУ, 2003. - 256 с.

32. Кремер Н.Ш. Математическая статистика. - М.: ЮРАЙТ, 2018. $-259 \mathrm{c}$.

33. Алексеев В.И. Прогнозирование динамики изменений орбитально-климатической диаграммы на основе методов вейвлетанализа и нечетких нейронных сетей // Вестник Югорского государственного университета. - 2018. - Т. 1 (48). - С. 13-21.

34. Weaver C.G. Fundamental causation: physics, metaphysics, and the deep structure of the world. - London: Routledge, 2018. $368 \mathrm{p}$.

35. Хименко В.И. Случайные данные: структура и анализ. - М.: ТЕХНОСФЕРА, 2017. - $424 \mathrm{c}$.

36. Бялко А.В. Палеоклимат: дополнения к теории Миланковича // Природа. - 2009. № 12. - С. 18-28.

37. Jose P.D. Sun's motion and sunspots // The Astronomical Journal. - 1965. - V. 70. - № 3. - P. 193-200.

38. Shirley J.H., Sperber K.R., Fairbridge R.W. Sun's interval and Luminosity // Solar Physics. - 1990. - V. 127. - № 2. - P. 379-392.

39. Darwin G.H. On the precession of a viscous spheroid and on the remote history of the Earth // Proc. of the Royal Society of London. - 1879. - V. 170. - P. 447-530.

40. Darwin G.H. On the secular changes in the elements of the orbit of a satellite revolving about a tidally distorted planet // Proc. of the Royal Society of London. - 1880. -V. 171. - P. 713-891.

41. Sidorenkov N.S. The interaction between Earth's rotation and geophysical processes. - Weinheim: WILEY-VCH Verlag GmbH and Co. KGaA, 2009. - 317 p.

42. Khlystov A., Dolgachov V.P., Domozhilova L.M. Barycentric movement of the Sun and Solar-Terrestrial relations // Biophysics. - 1992. - V. 37. - № 3. - P. 455-460.

43. Charvatova I. On the relation between solar motion and the solar activity in the years 1730-80 and 1910-60 a.d. // Bulletin Astronomic Institute Czech Republic. - 1990. - V. 41. - № 3. P. 200-204.

44. Прокудина В.С. Приложение метода планетных конфигураций к активным явлениям на Солнце // Сообщение Государственного Астрономического Института им. П.К. Штенберга. - 1973. - № 181. - C. 11-29.

45. Fairbridge R.W., Shirley J.H. Prolonged minima and the 179-yr cycle of the solar inertial motion // Solar Physics. - 1987. V. 110. - № 1. - P. 191-220.

46. Wood R.M., Wood K.D. Solar motion and sunspot comparison // Nature. - 1965. - V. 208. - № 5006. - P. 129-131.

47. The 90day oscillation of Jupiter's Great Red Spot revisited / J.M. Trigo-Rodrgues, A. Sanchez-Lavega, J.M. Gomez, J. Lecacheux, F. Colas, I. Miyazaki // 31st Annual Meeting of the DPS. Kursaal Centr. Poster, 1999.

48. Dickey J.O. Earth rotation // Global Earth Physics: a handbook of physical constants American Geophysical Union / Ed. by T.J. Ahrens. - Washington: American Geophysical Union, 1995. P. $356-368$

Поступила 16.06.2020 2.

\section{Информация об авторах}

Алексеев В.И., доктор технических наук, профессор Института цифровой экономики Югорского государственного университета. 
UDC 517.9; 004; 551.58

\title{
STUDYING CHANGES IN GLOBAL CLIMATE AS A COMPLEX SYSTEM USING WAVELET PHASE-FREQUENCY FUNCTIONS, PHASE-FREQUENCY AND PHASE-TEMPORAL CHARACTERISTICS OF HELOCOSMIC AND CLIMATIC VARIABLES. PART 1
}

\author{
Valery I. Alekseev, \\ v_alekseev@ugrasu.ru \\ Yugra State University, \\ 16, Chekhov street, Khanty-Mansiysk, 628012, Russia.
}

\begin{abstract}
The relevance of the research is caused by the need to establish the true causes and patterns of global climate change on Earth. The aim of the research is to develop and propose an effective method for extracting information on the variability of variables contained in the initial data of changes in heliocosmic and climate variables specified in the form of time series and apply this method to substantiate climatic changes on Earth.

Objects: time series of changes in heliocosmic and climate variables.

Methods: continuous direct wavelet transform of source data using a complex wavelet with the calculation of their time wavelet phasefrequency functions, phase-frequency and phase-time characteristics; evaluation of the frequency spectrum of functions on the phase-time characteristics of variables; assessment and analysis of density distribution of phase-frequency and phase-time characteristics; evaluation and analysis of correlation coefficients between the phase-frequency and phase-time characteristics of variables in equal time intervals.

Results. The author has obtained the wavelet time phase-frequency functions and their images, which allow visualizing and estimating the periodicity spectra of the studied variables. Estimates are obtained for the wavelet phase-frequency and phase-time characteristics of the variables, which make it possible to carry out the full range of comparative studies of the variables and to establish the patterns of their changes. These studies include: estimating the densities of distributions of these characteristics, which allow identifying the factors influencing these changes; calculation of correlation matrices between wavelet phase-frequency and phase-time characteristics of variables in specified equal time intervals, which allows assessing the degree of consistency of changes in variables in the phasefrequency domain and in the area of phase-time changes. A close relationship was established between the oscillatory processes of heliocosmic variables, solar activity and volcanic processes on Earth, and changes in carbon dioxide concentration in the atmosphere. It is shown that the movement of the Sun relative to the barycenter, caused by the movement of the giant planets of the Solar System, controls solar activity, tectonic processes on the Earth, including volcanic eruptions, the climate on the Earth
\end{abstract}

\section{Key words:}

Global climate change, continuous direct wavelet transform, wavelet time phase-frequency function, wavelet phase-frequency and phase time characteristics, correlation coefficients.

\section{REFERENCES}

1. Signals and consequences of climate change persist in 2018. Press release of the World Meteorological Organization. Document number 29112018. Date of publication November 29, 2018 Available at: https://public.wmo.int/en/media/press-releases / signals (accessed 20 May 2019).

2. Globalnoe poteplenie na $1,5{ }^{\circ} \mathrm{C}$. Doklad mezhpravitelstvennoy gruppy ekspertov po izmeneniyu klimata [Global warming by 1,5. Report of the Intergovernmental Panel on Climate Change (IPCC)] Available at: https://pop-science.ru/doklad-mgeik-2018-goda (accessed 20 May 2019)

3. Itogi konferentsii po izmeneniyu klimata v Katovitse SOP24 [Results of the conference on climate change in Katowice SOP24] Poland, 2018. Publication date 12/15/2018. Available at: http://ecounion.kz/?p=716 (accessed 20 May 2019).

4. Doklad ob ocobennostyakh klimata na territorii Rossiyskoy Federatsii za 2018 god [Report on climate features in the Russian Federation for 2018]. Moscow, 2019. 79 p. Available at: http://meteo.ru/events/102-raznoe/754-doklad-ob-osobennostyakhklimata-na-territorii-rossijskoj-federatsii-za-2018-god (accessed 20 May 2019).

5. Doklad o klimaticheskikh riskakh na territorii Rossiyskoy Federatsii [Report on climate risks in the Russian Federation]. Federalnaya sluzhba po gidrometeorologii i monitoring okruzhayuschey sredy [Federal Service for Hydrometeorology and Environmental Monitoring (ROSHYDROMET). Climatic center]. St. Petersburg, 2017. 106 p. Available at: http: //cc.voeikovmgo.ru/images/ dokumenty/2017/riski.pdf (accessed 20 May 2019).

6. Rosenzweig R. Global climate change policy and carbon markets. London, Spriger, 2016. $322 \mathrm{p}$

7. Carbon markets for greenhouse gas emission reduction in a warming world an evaluation of the world bank group's support to car bon finance. International Bank for Reconstruction and Development. Washington, 2018. $227 \mathrm{p}$.

8. Wang S., Lee W., Son Y. Low carbon development pathways in Indian agriculture. Change Adaptation Socio-Ecological Systems, 2017, vol. 3. pp. 18-26.

9. Gordon E., Lieberman B. Climate change in human history: prehistory to the present. London, Bloomsbury Academic \& Professional, 2018. $244 \mathrm{p}$.

10. Sovremennye globalnye izmeneniya prirodnoy sredy [Modern global changes in the environment]. Eds. R.K Klige., S.A. Lukyanova. Moscow, Nauchny mir Publ., 2006. Vol. 1, 696 p.

11. Sovremennye globalnye izmeneniya prirodnoy sredy [Modern global changes in the environment]. Eds. R.K. Klige S.A., Lukyanova. Moscow, Nauchny mir Publ., 2006. Vol. 2, 776 p.

12. Sovremennye globalnye izmeneniya prirodnoy sredy [Modern global changes in the environment]. T. 3. Faktory globalnykh izmeneni [Factors of global change] Eds. R.K. Klige, A.I. Khlystova. Moscow, Nauchny mir Publ., 2012. 444 p.

13. Khlystov A.I., Klige R.K., Sidorenkov N.S. Rezonans tretego roda $\mathrm{v}$ Solnechnoy sisteme [Resonance of the third kind in the solar system]. Sovremennye globalnye izmeneniya prirodnoy sredy [Modern global changes in the natural environment]. T. 3. Faktory globalnykh izmeneni [Factors of global change]. Moscow, Nauchny mir Publ., 2012. pp. 147-160.

14. Wilson Jan R.G. Izmenenie vo vrashchenii Zemli otnositelno baritsentra i klimaticheskiy effekt [Change in the Earth's rotation relative to the barycenter and the climatic effect]. Sovremennye globalnye izmeneniya prirodnoy sredy [Modern global changes in the natural environment]. T. 3. Faktory globalnykh izmeneni [Factors of global change]. Moscow, Nauchny mir Publ., 2012. pp. 78-102.

15. Khlystov A.I., Dolganov V.P., Domozhilova L.M. Baritsentricheskoe dvizhenie Solntsa i ego sledstviya dlya Solnechnor sistemy [The barycentric movement of the sun and its consequences for the 
solar system]. Sovremennye globalnye izmeneniya prirodnoy sredy [Modern global changes in the natural environment]. T. 3. Faktory globalnykh izmeneni [Factors of global change]. Moscow, Nauchny mir Publ., 2012. pp. 62-77.

16. Khlystov A.I., Somov B.V. Nelineyny metod prognoza 24-go solnechnogo tsikla [Nonlinear method for predicting the $24^{\text {th }}$ solar cycle]. Sovremennye globalnye izmeneniya prirodnoy sredy [Modern global changes in the natural environment]. T. 3. Faktory globalnykh izmeneni [Factors of global change]. Moscow, Nauchny mir Publ., 2012. pp. 137-146.

17. Sidorenkov N.S. Nestabilnosti vrashcheniya Zemli i globalnye izmeneniya prirodnykh protsessov [Earth rotation instabilities and global changes in natural processes]. Sovremennye globalnye izmeneniya prirodnoy sredy [Modern global changes in the natural environment]. Moscow, Nauchny mir Publ., 2006. Vol. 2, pp. 737-747.

18. Kasimov N.S., Klige R.K. Vvedenie. Problemy globalnykh izmeneniy [Introduction. Problems of global changes]. Sovremennye globalnye izmeneniya prirodnoy sredy [Modern global changes in the natural environment]. T. 3. Faktory globalnykh izmeneniy [Factors of global change]. Moscow, Nauchny mir Publ., 2012. pp. 19-34.

19. Miletsky E.V., Nagovitsyn Yu.A. Magnetic fields of sunspots in 21-22 cycles of solar activity. Bulletin of the State Astronomical Observatory, 2000, vol. 215, pp. 259-270. In Rus.

20. Volkano eruptions of 2014 and all eruptions since 1600 to present. The Big Wobble. June 18, 2016. Available at: http://www.thebigwobble.org/2016/06/volcano-eruptions-of-2014and-all.html1 (accessed 20 May 2019).

21. Khain V.E., Khalilov E.N. Tsiklichnost geodinamicheskikh protsessov: ee vozmozhnaya priroda [Cyclicity of geodynamic processes: its possible nature]. Moscow, Nauchny mir Publ., 2009. $520 \mathrm{p}$.

22. The growth of natural disasters over the past century according to the Center for Disaster Development Research (CRED). Available at: https://www: liveinternet.ru/users/famar/post367117884. (accessed 20 May 2019).

23. Gladilshchikova A.A., Semenov S.M. Intergovernmental Panel on Climate Change (IPCC): sixth assessment report cycle. Fundamental and Applied Climatology, 2017, vol. 2, pp. 13-25. In Rus.

24. Hurrell J. National Center for Atmospheric Research (NCAR) 2007. Available at: http://www. cgd.ucar.edu/cas/jhurrel/Data/ naodjfmindex.asc (accessed 20 May 2019).

25. Alekseev V.I. Weyvletny phazo-chastotny analiz klimaticheskikh peremennykh po dannym glybokogo burenia lednikovykh skvazhin v Antarktide [Wavelet phase-frequency analysis of climatic variables according to deep drilling of ice wells in Antarctica] Dinamika okpuzhayuschey sredy $i$ globalnye izmenenia klimata, 2013, vol. 4, no. 2, pp. 9-26.

26. Alekseev V.I. Weyvlet-analiz dinamiki izmeneniy yavlenia ElNino i ego prognozirovanie [Wavelet analysis of the dynamics of changes in the El Nino phenomenon and its forecasting]. Vestnik Yugorskogo gosudarstvennogo universiteta, 2018, vol. 3 (50), pp. 75-87.

27. Blatter K. Veyvlet-analiz. Osnovy teorii [Wavelet Analysis. Fundamentals of the theory]. Moscow, TEKHNOSFERA Publ., 2004. $280 \mathrm{p}$.

28. Dyakonov V.P. Veyvlety. Ot teorii $k$ praktike [Wavelets. From theory to practice]. Moscow, SOLON-Press, 2004. $400 \mathrm{p}$.

29. Malla S. Veyvlety v obrabotke signalov [Wavelets in signal processing]. Translated from English. Moscow, Mir Publ., 2005. $671 \mathrm{p}$.

Information about the authors

Alekseev V.I., Dr. Sc., professor, Yugra State University.
30. Moon F.C. Chaotic vibration. An introduction for applied scientists and engineers. Ithaca, New York, Theoretical and Applied Mechanics Cornel University, 1990. 312 p.

31. Bolshakov V.A. Novaya kontseptsiya orbitalnoy teorii paleoklimata [A new concept of the orbital theory of paleoclimate]. Moscow, MGU Publ., 2003. $256 \mathrm{p}$.

32. Kremer N.Sh. Matematicheskaya statistika [Mathematical statistics]. Moscow, Yurayt Publ., 2018. 259 p.

33. Alekseev V.I. Prognozirovanie dinamiki izmeneniy orbitalnoklimaticheskoy diagrammy na osnove metodov weyvlet-analiza i nechetkikh neyronnykh setetey [Predicting the dynamics of changes in the orbital-climatic diagram based on the methods of wavelet analysis and fuzzy neural networks]. Vestnik Yugorskogo gosudarstvennogo universiteta, 2018, vol. 1 (48), pp. 1-21.

34. Weaver C.G. Fundamental causation: physics, metaphysics, and the deep structure of the world. London, Routledge, 2018. 368 p.

35. Khimenko V.I. Sluchaynye dannye: struktura $i$ analiz [Random data: structure and analysis]. Moscow, Tekhnosfera Publ., 2017. $424 \mathrm{p}$

36. Byalko A.V. Paleoclimate: additions to the theory of Milankovitch. Nature, 2009, no. 12, pp. 18-28. In Rus.

37. Jose P.D. Sun`s motion and sunspots. The Astronomical Journal, 1965, vol. 70, no. 3, pp. 193-200.

38. Shirley J.H., Sperber K.R., Fairbridge R.W. Sun's interval and Luminosity. Solar Physics, 1990, vol. 127, no. 2, pp. 379-392.

39. Darwin G.H. On the precession of a viscous spheroid and on the remote history of the Earth. Proc. of the Royal Society of London, 1879, vol. 170 , pp. $447-530$

40. Darwin G.H. On the secular changes in the elements of the orbit of a satellite revolving about a tidally distorted planet. Proc. of the Royal Society of London, 1880, vol. 171, pp. 713-891.

41. Sidorenkov N.S. The interaction between Earth's rotation and geophysical processes. Weinheim, WILEY-VCH Verlag GmbH and Co. KGaA, 2009. 317 p.

42. Khlystov A., Dolgachov V.P., Domozhilova L., M. Barycentric movement of the Sun and Solar-Terrestrial relations. Biophysics, 1992, vol. 37, no. 3, pp. 455-460.

43. Charvatova I. On the relation between solar motion and the solar activity in the years 1730-80 and 1910-60 a.d. Bulletin Astronomic Institute Czech Republic, 1990, vol. 41, no. 3, pp. 200-204.

44. Prokudina V.S. Application of the method of planetary configurations to active phenomena on the Sun. Communication of the State Astronomical Institute named after P.K. Stenberg, 1973, no. 181, pp. 11-29. In Rus.

45. Fairbridge R.W., Shirley J.H. Prolonged minima and the 179 -yr cycle of the solar inertial motion. Solar Physics, 1987, vol. 110, no. 1, pp. 191-220.

46. Wood R.M., Wood K.D. Solar motion and sunspot comparison. Nature, 1965 , vol. 208 , no. 5006 , pp. 129-131.

47. Trigo-Rodrigues J.M., Sanchez-Lavega A., Gomez J.M., Lecacheux J., Colas F., Miyazaki I. The 90day oscillation of Jupiter's Great Red Spot revisited. Journal Planetary and Space Science, 2000, vol. 48, Iss. 4, pp. 331-339.

48. Dickey J.O. Earth rotation. Global Earth Physics: a handbook of physical constants American Geophysical Union. Ed. by T.J. Ahrens. Washington, American Geophysical Union, 1995. pp. 356-368.

Received: 16 June 2020. 